\title{
Cooling of 3D granular gases in microgravity experiments
}

\author{
Kirsten Harth ${ }^{1,2, \star}$, Torsten Trittel ${ }^{1}$, Sandra Wegner ${ }^{1}$, and Ralf Stannarius ${ }^{1, \star \star}$ \\ ${ }^{1}$ Institute of Experimental Physics and MARS, Otto von Guericke University, Magdeburg, Germany \\ ${ }^{2}$ current address: Physics of Fluids, University of Twente, Enschede, The Netherlands
}

\begin{abstract}
Granular gases are a most peculiar state, superficially similar to molecular gases: They are loose ensembles of moving grains, rarely interacting with each other and with container walls. The most investigated scenario is the "granular cooling", the collective loss of energy from an initially excited state. We present an experimental study of the cooling of a 3D granular gas of rodlike grains in micro-gravity. Driven steady states of non-spherical grains are characterized by a lack of energy equipartition between the degrees of freedom of translation and rotation. Excitation by vibrating walls additionally introduces strong gradients in the direction and magnitude of translational velocities. We show that the degrees of freedom equilibrate during granular cooling in the homogeneous cooling state. The energy loss follows a $t^{-2}$ scaling. In addition, the alignment of the rod axes with the excitation direction and with the instantaneous velocities are altered during this process.
\end{abstract}

Excited granular materials are frequently encountered in nature, from dust storms to the Saturn rings. Many technological processes involve transport and handling of granular materials, often also moving loose or compact granular matter. However, an appropriate modeling remains challenging, not only due to insufficient computational resources to deal with most of these many-particle systems, but also due to the large range of scales involved in continuum models which have to capture the range of container to contact dimensions. Collisions, packing and jamming of the grains result in essential differences between granular and fluid flows, e.g. clogging and intermittent flows of hoppers or the occurrence of stick-slip events. The grain size distributions and shapes may influence the phenomena tremendously, e.g. via alignment [1] or secondary flow [2] of anisometric grains.

Ensembles of grains in which collisions almost exclusively involve pairs of particles are comparatively simple. In the extremely dilute case of granular gases with grain volume fractions of only few percent, individual collisions are directly accessible in experiments, and the influence of grain properties on the ensemble behavior can be studied. In addition, granular gases represent one of the simplest non-equilibrium many particle systems in statistical mechanics. The main difference to molecular gases lies in the dissipation of mechanical energy during collisions. This fact motivated various theoretical and numerical studies in the literature. Experimental validation is scarce, primarily due to the poor compatibility of favorable theoretical conditions (e.g. large number of particles, periodic boundary conditions, random energy supply in driven systems) with experimental realizability.

\footnotetext{
^e-mail: kirsten.harth@ovgu.de

${ }^{\star}$ e-mail: ralf.stannarius@ovgu.de
}

One of the most intriguing and most addressed aspects of granular gas dynamics is the loss of kinetic energy from an initially homogeneous agitated state, the "granular cooling". Related to it are the formation of planetesimals, the evolution of planetary rings, or the dynamics of objects in the asteroid belt. Haff [3] described the energy loss in a spatially homogeneous granular gas of frictionless spherical grains: The kinetic energy decays with time $t$ as

$$
E_{\mathrm{kin}}(t)=\frac{E_{\mathrm{kin}}(t=0)}{(1+t / \tau)^{2}} \propto t^{-2}(t \rightarrow \infty),
$$

where the characteristic dissipation rate

$$
1 / \tau=2\left(1-\epsilon^{2}\right) \cdot \overline{\mid \vec{v}} \mid / \lambda
$$

depends on the normal coefficient of restitution $\epsilon$, the mean free path $\lambda$ and the mean absolute velocity $\overline{|\vec{v}|}$. All translational degrees of freedom (DOF) are treated equivalently there, rotations are not included. Haff simplifies in that the squared mean absolute velocity is set equal to the mean squared velocity. With proceeding energy decay, density inhomogeneities and clusters can develop in the system, first demonstrated in a 2D simulation [4] and observed in a small ensemble of levitated grains [5]. Depending on the grain shape [6,7] or for finite system size [8], those clusters may be unstable, they can breakup and dissolve. Simulations of ensembles of elongated grains predict a loss of the equipartition of kinetic energies in general $[6,7,9,10]$. It is a priori unclear whether Haff's prediction will also apply to a granular gas of (frictional) rodlike grains. The models in the literature do not provide unique answers $[6,10]$.

Experimental realization of a homogeneously cooling granular gas is challenging: The initial excitation of the 
grains generally requires vibrations of the container or its walls. An alternative magnetic excitation scheme was recently developed to provide contactless forcing in the bulk [11]. For two-dimensional granular gases, the interaction with the substrate must be taken into account [12]. One way out is levitation by electromagnetic fields $[5,13]$ or by flow $[14,15]$. The realization of $3 \mathrm{D}$ granular gases in low external excitation requires microgravity. The first study by Falcon et al. investigated continuously driven ensembles of spheres and revealed dynamic clustering [16], which was investigated in detail and modeled in Refs. $[17,18]$. Afterwards, mainly 2D systems of few particles were studied [19, 20]. Maaß et al. [5] and Weidling et al. [21] present results for small ensembles of highly dissipative grains during granular cooling, obtained from 2D perspective views.

A spatially homogeneous grain distribution in a steady state can be achieved with rodlike grains [22-24]. An advantage of rods over spheres is the reduction of the mean free path at equal volume fraction of particles and the trackability of rotations. In a steady excited state, we found an excess of kinetic energy of translational compared to rotational degrees of freedom from a $2 \mathrm{D}$ perspective evaluation of the 3D granular gas [23]. Velocity distributions in the coordinates $i \in\{x, y, z\}$ are non-Gaussian, rather $p\left(v_{i}\right) \propto \exp \left(-\left|v_{i}\right|^{1.5}\right)$. The direction of the vibrating walls $(x)$ is distinguished by the highest kinetic energy and by exponential high-velocity tails. We recently improved the setup and developed methods for preparing and obtaining 3D data of granular gases in few seconds. With a stereo camera setup, rods become trackable in 3D. This allows 3D experiments using drop tower shots at the ZARM Bremen [24], offering $\approx 9 \mathrm{~s}$ of excellent micro-gravity. Parabolic flight experiments are inappropriate for low-excitation experiments in general [24] due to the $g$-jitter, but may be applied for investigation at strong excitation [18] or of dense granular systems [25].

This presentation will provide the first detailed experimental study of free homogeneous cooling of a 3D granular gas. Our setup is similar to that of Refs. [22, 24]: It consists of a cuboid container of $\approx 11 \times 8 \times 8 \mathrm{~cm}^{3}$ with two movable side walls. Front and top walls are made of ITO coated acrylic glass, other walls are flat white painted aluminum. Front and top view are recorded using two GoPro cameras with c-mount objectives at 100 frames per second, $1280 \times 960 \mathrm{pixel}^{2}$. Our granular gases consist of 374 colored insulated copper wire pieces with $\varnothing \approx 1.35 \mathrm{~mm}$, length $\ell=10 \mathrm{~mm}$, corresponding to a volume fraction of $0.76 \%$ and an estimated mean free path of $2.2 \mathrm{~mm}$ [27]. Here we restrict to an average over 3 experiment runs, additional data are currently being evaluated and published elsewhere. The mass of a rod is $m=0.0375 \mathrm{~g}$, and moments of inertia for a rotation axis parallel and perpendicular to the symmetry axis are $J_{\|}=4.63 \cdot 10^{-12} \mathrm{~kg} \mathrm{~m}^{2}$ and $J_{\perp}=315 \cdot 10^{-12} \mathrm{~kg} \mathrm{~m}^{2}$, respectively.

The initial state is prepared by injection of kinetic energy with the vibrating walls at an amplitude $A \approx 6 \mathrm{~mm}$

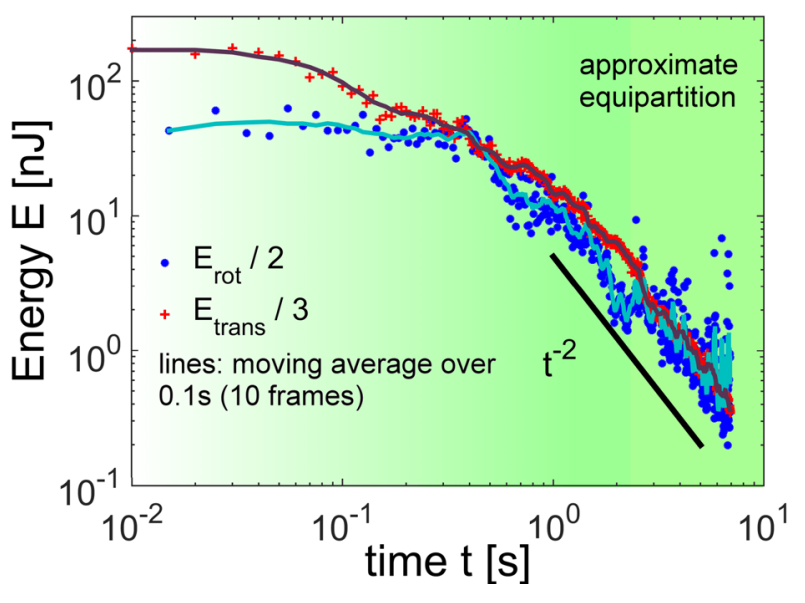

Figure 1. Decay of the translational and rotational kinetic energies per rod and DOF: The initial excess energy in translational over rotational DOF equilibrates within $\approx 2 \ldots 2.5 \mathrm{~s}$, to reach approximately equipartition of kinetic energy.

(peak-peak) and frequency $f=30 \mathrm{~Hz}$ for $1 \ldots 1.5 \mathrm{~s}$. Data are averaged over all experiment runs. Colored grains are tracked in each perspective by a semi-automatic custom routine. 3D data are calculated using the camera calibration toolbox [26] supplemented by a custom routine. The experimental uncertainty of positions and orientations depends on the grain orientation in both perspectives and the overlap with other rods, it is maximal e. g. when the rod makes only a small angle to the $z$-axis. We plot the timedependent positions (center of mass) and angles to the coordinate axes for each rod and identify segments of undisturbed motion of each rod manually. The resulting segments of center of mass translations in each coordinate are fitted by linear functions. For rotations, we compute the reorientation angle in a time step from the vector product of initial and final orientation, then divide by the respective time step (may be larger than 1 frame). After that, we discard obvious detection errors and average all values of the rotational velocity obtained in a time sequence between rod collisions (as for the translational velocities). As the two degrees of freedom corresponding to rotations around axes perpendicular to the long rod axis cannot be distinguished, we consider only the mean squared rotational velocities of both degrees of freedom together, $\omega_{\perp}$. We expect the third rotational DOF (around the long particle axis) to be much less excited as it is only excited via friction during rod-rod collisions, but it has not been measured here quantitatively. Fitting is especially important during the second half of our measurement time and for the rotational velocities, to minimize the influence of detection noise. On average, the rod orientations possess larger errors than the center of mass positions. We excluded rods with positions less than $1 \mathrm{~cm}$ from the walls in this evaluation, they are assumed to be not equilibrated with the bulk due to interactions with the container walls.

Figure 1 shows the decay of the translational and the rotational energy per rod and degree of freedom over the complete accessible time of $\approx 7 \mathrm{~s}$. In the driven 


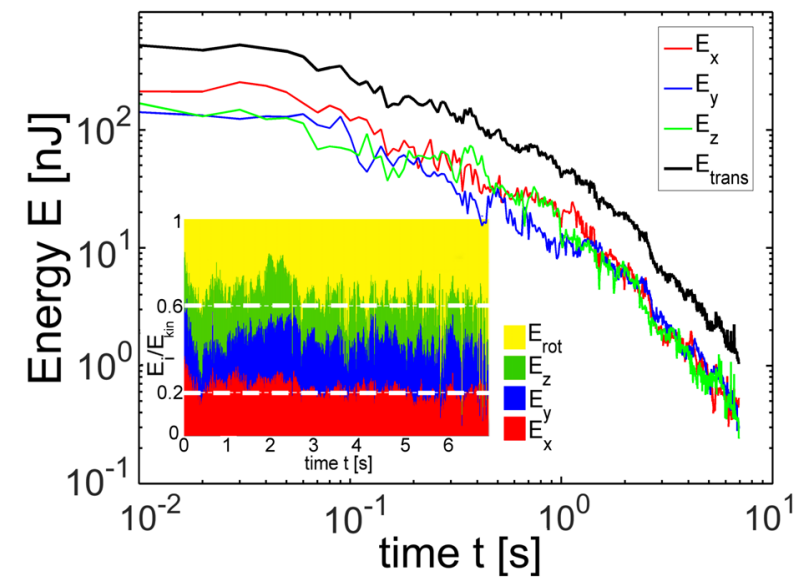

Figure 2. Translational kinetic energy per particle and DOF in the directions of the box coordinate system, where $x$ is the direction enclosed by the vibrating walls, $y$ and $z$ by non-moving walls. Inset: Instantaneous fraction of the kinetic energy per DOF and the rotational energy (2 DOF). Dashed lines indicate $1 / 5 E_{\text {kin }}(t)$ and $3 / 5 E_{\text {kin }}(t)$.

steady state, equipartition is violated as expected from Refs. [9, 10, 23]: Initially, the mean translational energy per DOF is $\approx 180 \mathrm{~nJ}$, whereas the rotational energy per DOF is $\approx 40 \mathrm{~nJ}$, less than one quarter of the translational kinetic energy. Both values strongly approach each other during the first $0.2 \mathrm{~s}$ of cooling. Long-term deviations from an equilibrated state are observed until $t \approx 2 \ldots 2.5 \mathrm{~s}$. After this, the DOF equilibrate within experimental noise. Then, both translational and rotational kinetic energy decay $\propto t^{-2}$, as predicted by Haff [3] and in qualitative agreement with Refs. [10]. In particular, this is in contrast to the $2 \mathrm{D}$ simulations of elongated viscoelastic particles by Kanzaki et al. [6], who predicted differing power laws for translational and rotational DOF.

The initial bias in the energy partition must be attributed to the external driving of the system. The direction that is directly excited by shaking walls is distinguished by a higher initial kinetic energy than the indirectly excited DOF, see Fig. 2. The bias is larger than in our previous steady state experiments [23], probably due to the stronger excitation at approximately equal mean free paths. In particular, the velocity distributions in excitation direction $x$ differ depending on the distance to the walls, as discussed in Refs. [20, 27]. Thus, the assumption of a spatially homogeneous initial state is not in accordance with the velocity field expected in this experiment. In the inset of Fig. 2, we display the fractions of the mean total energy per rod for the different DOF. We observe a pronounced transfer from all translational DOF to the rotational DOF during the first $0.5 \mathrm{~s}$ of cooling, followed $\mathrm{b}$ a slight growth in translational energy until $t \approx 2.5 \mathrm{~s}$. These transient deviations from equipartition are also clearly identified in Fig. 1. Equilibration (within the accuracy of the current data) of the three translational degrees of freedom occurs on a similar time scale as the equilibration of translational and transverse rotational degrees of freedom. Rotations
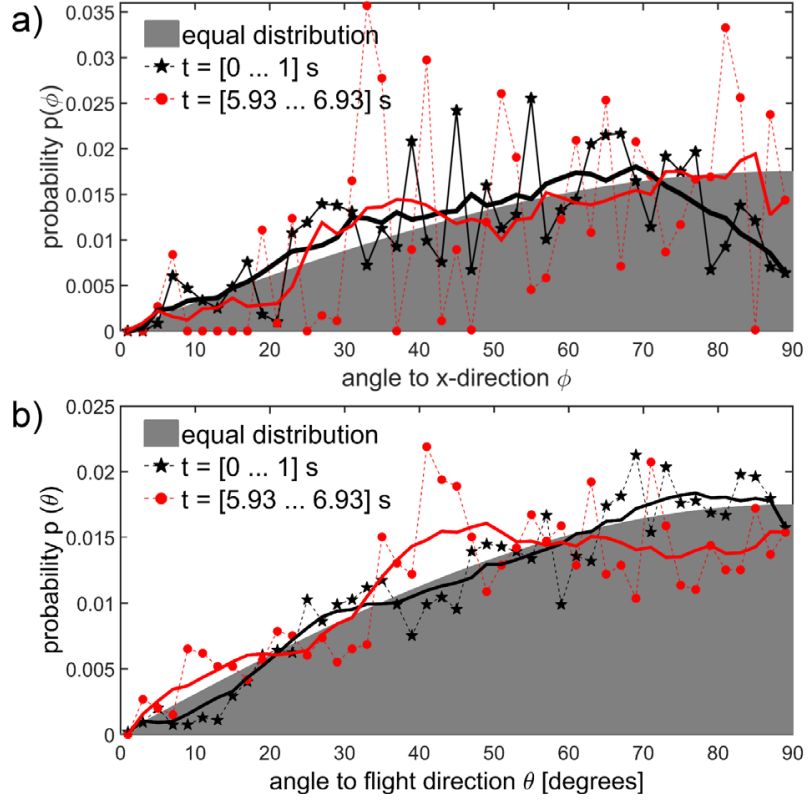

Figure 3. Distributions of the angles of the long axes of the rods respective to a) the direction of wall excitation $x$, and b) the current flight direction, during the initial and last second of the measurement. Grey areas represent equally distributed orientations.

around the symmetry axis are only excited via friction, and not measured here.

Finally, let us consider the distribution of orientations of the rods in order to characterize differences in the initial and final states in more detail. Fig. 3a) shows the distribution of the angles $\phi$ between of the rods' long axes and the excitation direction $x$, and $\mathrm{b}$ ) shows the distribution of the angles $\theta$ of the long axes respective to the momentary flight direction. Comparing the first and the last seconds to a random distribution, one finds initially a decreased probability of rod motion perpendicular the $x$ direction. This feature is caused by the strong excitation by the walls, it reflects the incomplete equilibration of the directions of motion via collisions in he excited state. Wright et al. [28] studied rods bouncing onto a vibrating wall. They reported consistently lower probabilities of impacts with the long axis parallel to the wall, but provided no corresponding data after rebound. Our recent results on energy input and distribution of rods rebounding from a sinusoidally vibrating wall reveal a strong excess of translational energy in direction of vibration over the injected rotational energy and translational kinetic energy of the other DOF [29]. This bias vanishes rapidly during granular cooling. Obviously, the collisions with the non-moving walls introduce only negligible bias (if any at all).

A related question is whether the permanent collisions in absence of external excitation influence the orientational distribution of the rods respective to their direction of motion. For rough spherical grains, correlations between the axis of rotations and translations were reported in the literature [30]. We find clear evidence of a preferential alignment in Fig. 3b: Initially, the rods 
translate in arbitrary direction respective to their long axis. However, as the excitation bias ends, partial alignment occurs, evident in the last second of our measurement. The scattering cross section of a rotating rod is maximized when it is directed perpendicular to its current direction of motion (propeller-like, $\vec{v} \| \vec{\omega}$ ). This results in higher collision rates for these rods compared to those with smaller angles $\theta$ between the rod symmetry axis and $\vec{v}$. Note the difference to Ref. [14], where the preferential motion along the rod axis is due to the high area fraction.

Summarizing, we present the first 3D experimental analysis of a freely cooling granular gas on the particle level. During the $7 \mathrm{~s}$ duration of the measurement, the kinetic energy decays by more than 2 orders of magnitude. We do not observe significant density inhomogeneities. While the grain distribution is initially close to homogeneous, the velocity fields include strong gradients especially in excitation direction, and energy equipartition is violated. After the excitation is stopped, we observe an equilibration of all DOF within statistical noise during the first $\approx 2.5 \mathrm{~s}$ of granular cooling. The full evaluated data sets will provide more detailed insights into the cooling process and its influence, e.g. on the shape of velocity distributions or spatial inhomogeneities. In direct consequence, it will be of interest to extend the observation time to search for a potential onset of spatial inhomogeneities or clustering of grains, a corresponding experiment is scheduled for a sounding rocket flight in 2017. Investigation of the energy partition between different components of particle mixtures provide a perspective to yield more insight into the ensemble dynamics of polydisperse or mixed shape systems.

\section{Acknowledgements}

The authors acknowledge funding by the German Aerospace Center DLR, projects 50WM1241 and 50WM1344, and by the German Science Foundation (DFG) Grant STA 425/34-1. We cordially thank the ZARM staff in Bremen for their excellent support in performing the drop tower experiments.

\section{References}

[1] e.g. T. Börzsönyi et al., Phys. Rev. Lett. 108228302 (2012); Phys. Rev. E 86051304 (2012); New J. Phys. 18093017 (2016)

[2] G. Wortel et al., Soft Matter 112570 (2015); D. Fischer et al., New J. Phys. 18113006 (2016)

[3] P. K. Haff, J. Fluid Mech. 134401 (1983)

[4] I. Goldhirsch and G. Zanetti, Phys. Rev. Lett. 701619 (1993)
[5] C. C. Maaß et al., Phys. Rev. Lett. 100248001 (2008)

[6] T. Kanzaki, R. C. Hidalgo, D. Maza, and I. Pagonabarraga, J. Stat. Mech. 2010 P06020 (2010)

[7] M. Huthmann, T. Aspelmeier, and A. Zippelius, Phys. Rev. E 60654 (1999)

[8] M. Hummel and M. G. Mazza, Phys. Rev. E 93 022905 (2016)

[9] T. Aspelmeier, G. Giese, and A. Zippelius, PRE 57 857 (1998); G. Costantini et al., J. Chem. Phys. 122 164505 (2005)

[10] F. Villemot and J. Talbot, Gran. Matter 1491 (2012); S.M. Rubio-Largo, et al., Physica A 443477 (2016)

[11] M. Sperl, private communication, unpublished.

[12] J. S. van Zon et al., Phys. Rev. E 70040301 (2004)

[13] A. Snezhko, I. S. Aranson and W.-K. Kwok, Phys. Rev. Lett. 94108002 (2005); K. Kohlstedt et al., Phys. Rev. Lett. 95068001 (2005)

[14] L. J. Daniels et al., Phys. Rev. E 79041301 (2009)

[15] K. Nichol and K. E. Daniels, Phys. Rev. Lett. 108 018001 (2012); J. C. Burton et al., Phys. Rev. E 88 062204 (2013)

[16] E. Falcon et al., Phys. Rev. Lett. 83440 (1999)

[17] E. Opsomer, F. Ludewig, and N. Vandewalle, Phys. Rev. E 84051306 (2011)

[18] A. Sack et al., Phys. Rev. Lett. 111018001 (2013); J. Kollmer et al., Phys. Rev. Appl. 3024007 (2015); AIP Conf. Proc. 1542811 (2013)

[19] e.g. S. Tatsumi et al., J. Fluid Mech. 641521 (2009); Y. Grasselli, A. Bossis, and R. Morini, Eur. Phys. J. E 38 (2015)

[20] C. Yanpei et al., J. Physique 327 (2011)

[21] R. Weidling, C. G̈̈tler, and J. Blum, Icarus 218688 (2012)

[22] K. Harth et al., European Space Agency SP-700, 493 (2011)

[23] K. Harth et al., Phys. Rev. Lett. 110144102 (2012); AIP Conf. Proc. 1542807 (2013)

[24] K. Harth et al., Adv. Space Res. 551901 (2015)

[25] P. D. Yu, S. Frank-Richter, and M. Sperl, Gran. Matter 16165 (2014); N. Murdoch et al., Granular Matt. 15 129 (2013)

[26] Camera Calibration Toolbox by J.-Y. Bouguet, www.vision.caltech.edu/bouguetj/calib ${ }_{d} \mathrm{oc} /$

[27] K. Harth, T. Trittel and R. Stannarius, in preparation

[28] H. S. Wright, M. R. Swift, and P. J. King, Phys. Rev. E 74061309 (2006)

[29] T. Trittel, K. Harth, and R. Stannarius, under review (2017).

[30] M. Huthmann and A. Zippelius, Phys. Rev. E 56 R6275 (1997); N.V. Brilliantov et al., Phys. Rev. Lett. 98128001 (2007) 